\title{
Ocrelizumab: A Review in Multiple Sclerosis
}

\author{
Yvette N. Lamb ${ }^{1}$
}

Accepted: 12 January 2022 / Published online: 22 February 2022

(c) Springer Nature Switzerland AG 2022

\begin{abstract}
Ocrelizumab (Ocrevus $\left.{ }^{\circledR}\right)$ is an intravenously administered, humanized anti-CD20 monoclonal antibody approved for the treatment of adults with relapsing forms of multiple sclerosis (RMS) or primary progressive multiple sclerosis (PPMS). The efficacy of ocrelizumab in reducing relapse rates and disease activity in patients with RMS was demonstrated in pivotal trials (versus interferon $\beta$-1a) and supporting single-arm studies in specific subpopulations. In patients with PPMS, ocrelizumab reduced measures of clinical and MRI progression relative to placebo. Clinical benefits were maintained over $\geq 7.5$ study years of treatment. Ocrelizumab was generally well tolerated and no new safety signals have emerged with long-term use. Extensive (albeit short-term) real-world data pertaining to ocrelizumab is consistent with that from clinical trials. Ocrelizumab provides the convenience of short, half-yearly infusions. Ocrelizumab continues to represent a generally well-tolerated, high-efficacy disease-modifying therapy (DMT) for RMS and is a valuable treatment for delaying disease progression in patients with PPMS (for whom there are currently no other approved DMTs).
\end{abstract}

\section{Plain Language Summary}

Multiple sclerosis (MS) is a chronic, immune-mediated, neurodegenerative disease of the CNS. In most patients, it starts as relapsing-remitting MS (RRMS), which involves exacerbations of neurological symptoms (i.e. relapses) followed by periods of remission. In the less common primary progressive MS (PPMS), disability accrues steadily from disease onset. It is now understood that B cells play key roles in MS pathophysiology. Ocrelizumab (Ocrevus ${ }^{\circledR}$ ), a monoclonal antibody that selectively depletes CD20+ B cells, is approved for treating adults with RMS and PPMS in various countries worldwide. Ocrelizumab reduces relapse rates and indicators of disease activity in patients with RMS, and delays the worsening of disability in patients with RMS and PPMS. Of convenience to patients, ocrelizumab is intravenously administered every six months and can be infused rapidly (over $\approx 2$ hours) without its safety being substantially altered. Ocrelizumab is a generally well-tolerated and highly effective treatment option for RMS and constitutes the first approved pharmacotherapy for PPMS.

Digital Features for this Adis Drug Evaluation can be found at https://doi.org/10.6084/m9.figshare.17009108.

The manuscript was reviewed by: H.-P. Hartung, Department of Neurology, Heinrich-Heine University, Dusseldorf, Germany; Brain and Mind Center, University of Sydney, Sydney, Australia; F. Ladeira, Neurology Department, Hospital dos Capuchos, Centro Hospitalar Lisboa Central, Portugal.

Yvette N. Lamb

demail@ springer.com

1 Springer Nature, Mairangi Bay, Private Bag 65901, Auckland 0754, New Zealand

\section{Ocrelizumab: clinical considerations in MS}

Limits relapses, inflammatory activity and disease progression in patients with RMS

Delays clinical and MRI progression in patients with PPMS

Clinical benefits sustained over long-term treatment

Most common adverse events include infusion-related reactions and infections (mostly of mild to moderate severity) 


\section{Introduction}

Multiple sclerosis (MS), an immune-mediated neurodegenerative disease of the CNS, is characterized by inflammatory lesions, demyelinating plaques and, as the disease progresses, irreversible axonal damage [1]. The most prevalent phenotype at diagnosis is relapsing-remitting MS (RRMS; representing $\approx 85 \%$ of cases of MS), in which patients experience recurrent relapses and remissions of neurological symptoms [2]. Over time, RRMS transitions to a more steadily progressive disease [i.e. secondary progressive MS (SPMS)] in most untreated patients. Primary progressive MS (PPMS) is less common (representing $\approx 10$ to $20 \%$ of cases) and is characterized by a steady worsening of symptoms from disease onset, with no phases of remission [2].

Treatment of MS aims to reduce relapses and delay disease progression [3]. Long-term management utilizes disease-modifying therapies (DMTs), which typically exert therapeutic benefits through suppressing or modulating immune function and inflammatory responses [3, 4]. Potent DMTs include B cell-depleting monoclonal antibodies (mAbs) targeting the CD20 surface antigen, the clinical success of which has challenged the classical view of MS as a $\mathrm{T}$ cell-mediated disease and led to a new appreciation of the central roles of B cells in MS pathophysiology [4, 5]. In recent years, there has been a focus on the development of humanized or fully human B cell-depleting anti-CD20 mAbs for the treatment of MS [6]. These may offer reduced immunogenicity and greater potency relative to off-label chimeric anti-CD20 mAb pharmacotherapy [6].

Ocrelizumab (Ocrevus ${ }^{\circledR}$ ) is an intravenously administered, recombinant humanized anti-CD20 mAb approved for the treatment of relapsing forms of MS and PPMS in various countries worldwide (Sect. 4) [7, 8]. The use of ocrelizumab in these indications has been reviewed previously (in CNS Drugs [9]). The current article provides an updated review of the therapeutic efficacy and safety/tolerability of ocrelizumab in MS. An overview of the pharmacological properties of ocrelizumab is also provided (Table 1).

\section{Therapeutic Efficacy of Ocrelizumab}

The efficacy of ocrelizumab in the treatment of patients with relapsing MS (RMS) was evaluated in two pivotal, randomized, double-blind, double-dummy, active-controlled, multinational phase III trials (OPERA I and OPERA II) with identical protocols (Sect. 2.1.1) [10]. OPERA results are supported by interim data from an open-label, single-arm, multicentre, phase IV study in patients with active RMS (PRO-MSACTIVE; Sect. 2.1.2) [11], in addition to interim data from ongoing phase IIIb or IV trials in patients with treatment-naïve, early stage RRMS (ENSEMBLE [12]) and RMS previously treated with natalizumab (ENCORE [13]). The specific efficacy of ocrelizumab in patients with RRMS and a suboptimal response to prior DMTs has been investigated in two open-label, single-arm, multicentre phase IIIb studies (CHORDS in North America [14] and CASTING in the EU [15]; Sect. 2.1.3). The efficacy of ocrelizumab in the treatment of patients with PPMS was evaluated in the pivotal, randomized, double-blind, placebo-controlled, multinational, phase III ORATORIO trial (Sect. 2.2) [16]. Data on the real-world effectiveness of ocrelizumab are also available (Sect. 2.3).

\subsection{In Relapsing Multiple Sclerosis}

\subsubsection{Pivotal Trials}

OPERA I and II enrolled adults aged $18-55$ years with RMS (based on the 2010 revised McDonald criteria), an Expanded Disability Status Scale (EDSS) score of 0-5.5 at screening (scale ranges from 0 to 10 , with higher scores indicating greater disability), a history of documented clinical relapses (at least two in the previous 2 years or one in the year before screening), abnormalities consistent with MS in magnetic resonance imaging (MRI) of the brain, and no neurological worsening for $\geq 30$ days before screening and baseline [10]. Patients received ocrelizumab $600 \mathrm{mg}$ (every 24 weeks; administered as two $300 \mathrm{mg}$ infusions on days 1 and 15 for the initial dose and as single $600 \mathrm{mg}$ infusions thereafter) or subcutaneous interferon $\beta$-1a $44 \mu \mathrm{g}$ (administered three times each week) for 96 weeks. At baseline, the mean time since MS diagnosis was $\approx 4$ years in each trial. The majority of patients ( $\approx 75 \%$ overall) had not received any DMT in the 2 years prior to screening [10].

In both OPERA I and II, ocrelizumab significantly $(p<0.001)$ reduced the annualized relapse rate (ARR) relative to interferon $\beta$-1a at week 96 (Table 2; primary endpoint) [10]. The ARR was $46 \%$ lower with ocrelizumab versus interferon $\beta$-1a in OPERA I and $47 \%$ lower in OPERA II (Table 2) [10]. In the pooled OPERA I and II populations, the ARR improvement with ocrelizumab versus interferon $\beta$-1a was observed across prespecified subgroups based on age ( $<40$ vs $\geq 40$ years), sex, study region (USA vs other), baseline body mass index $\left(<25 \mathrm{~kg} / \mathrm{m}^{2}\right.$ vs $\geq 25 \mathrm{~kg} /$ $\mathrm{m}^{2}$ ), baseline EDSS ( $\left.<4 \mathrm{vs} \geq 4\right)$ and baseline gadoliniumenhancing $\mathrm{T}_{1}$ lesions ( $0 \mathrm{vs} \geq 1$ ); rate ratios were $0.36-0.74$ ( $p$-values $<0.05)$ in all subgroups, with the exception of patients aged $\geq 40$ years (rate ratio $0.76 ; p=0.073$ ) [17].

Various other measures of disease activity or progression were also improved with ocrelizumab versus interferon $\beta-1 \mathrm{a}$. In prespecifed pooled analyses of OPERA I and II data, ocrelizumab significantly ( $p \leq 0.02)$ improved the 


\section{Pharmacodynamic properties}

Precise mechanisms underlying therapeutic benefits of ocrelizumab in MS not fully elucidated; thought to involve immunomodulation through ocrelizumab binding to CD20 and reducing the number and function of $\mathrm{CD} 20^{+} \mathrm{B}$ cells $[7,8]$

Selectively depletes $\mathrm{CD} 20^{+}$B cells via antibody-dependent cellular cytotoxicity, complement-dependent cytotoxicity, antibody-dependent cellular phagocytosis, and apoptosis, while sparing the capacity for B cell reconstitution, pre-existing humoral immunity and innate immunity [8]

Reduced CD19+ $\mathrm{B}$ cells (surrogate marker for CD20 ${ }^{+} \mathrm{B}$ cell expression) in blood to negligible levels by 14 days post-infusion in pts with RMS and PPMS (B cell depletion sustained throughout treatment in 96\% of pts) [54]; median time to B cell repletion was 72 weeks (range 27-175 weeks) after stopping therapy in pts with RMS [54, 55]

Significantly $(p<0.05)$ reduced CSF B cells, CSF NfL and serum NfL from baseline to week 52 in pts with RMS [56] and CSF B cells in pts with PPMS [57] in a biomarker study (OBOE); CSF T cells were non-significantly reduced in each pt cohort at this timepoint [56, 57]

Reduced markers of active gliosis relative to interferon $\beta$-1a in pts with RMS [58]

\section{Pharmacokinetic properties}

Pharmacokinetic parameters as expected for an IgG1 monoclonal antibody [8, 54] and comparable across pts with RMS and PPMS [54] Overall exposure following a single $600 \mathrm{~mL}$ infusion did not differ from that following two $300 \mathrm{mg}$ infusions $[8,54]$

Estimated central volume of distribution $2.78 \mathrm{~L}$, peripheral volume $2.68 \mathrm{~L}$ and inter-compartment CL $0.29 \mathrm{~L} /$ day in PPK model [7, 8, 54]

Primarily cleared via catabolism [7, 8]; estimated constant CL 0.17 L/day and terminal elimination half-life 26 days [7, 8, 54]

Dosage adjustments based on liver or kidney function not expected to be necessary [8]

Based on limited pharmacokinetic data, no dosage adjustment required in pts $\geq 55$ years of age [8]

Interactions with drugs and vaccines

Potential additive immunosuppressive effects should be considered when coadministering ocrelizumab with other immunosuppressive therapies in the USA [7] and when initiating ocrelizumab after an immunosuppressive therapy (or vice versa) in the EU and USA [7, 8]; in the EU, coadministering ocrelizumab with other immunosuppressive therapies (except corticosteroids) is not recommended [8]

Pts with RMS receiving ocrelizumab mounted attenuated humoral responses to non-live vaccines (tetanus toxoid-containing vaccine, 23-valent pneumococcal polysaccharide vaccine, and seasonal influenza vaccines) and the keyhole limpet hemocyanin neoantigen in the phase IIIb VELOCE study $[7,8,59]$; in the EU, it is recommended that ocrelizumab recipients are vaccinated with inactivated seasonal influenza vaccines [8]; in the USA, non-live vaccines should be administered $\geq 2$ weeks prior to initiating ocrelizumab if possible [7]

Vaccination with live or live-attenuated vaccines is not recommended during treatment with ocrelizumab or after discontinuation but prior to B cell repletion [7, 8]; pt immunisation status should be reviewed and any immunisation (with live-attenuated or live vaccines in the USA) should be completed $\geq 4$ weeks (USA) or $\geq 6$ weeks (EU) prior to initiating ocrelizumab [7,8]

Due to potential for B cell depletion in infants of mothers exposed to ocrelizumab during pregnancy, live or live-attenuated vaccines should not be administered to infants until recovery $[7,8]$

$C S F$ cerebrospinal fluid, $C L$ clearance, $e G F R$ estimated glomerular filtration rate, $I g G 1$ immunoglobulin G1, $I V$ intravenous, $M S$ multiple sclerosis, $N f L$ neurofilament light chain, $P P K$ population pharmacokinetic, $P P M S$ primary progressive multiple sclerosis, $p t(s)$ patient(s), $R M S$ relapsing forms of multiple sclerosis

proportions of patients with disability progression confirmed at 12 weeks (12-week CDP) and 24 weeks (24-week CDP) and disability improvement confirmed at 12 weeks (12-week CDI; Table 2) [10]. With respect to MRI endpoints, ocrelizumab significantly $(p<0.001)$ reduced mean numbers of gadolinium-enhancing $\mathrm{T}_{1}$ lesions, new or enlarged hyperintense $T_{2}$ lesions (indicative of plaque formation), and new hypointense $T_{1}$ lesions relative to interferon $\beta$-1a in both trials (Table 2). While treatment groups did not significantly differ with respect to change in MS Functional Composite (MSFC) score from baseline to week 96 in OPERA I (first non-significant $p$-value in hierarchical testing procedure), a benefit of ocrelizumab over interferon $\beta$ - 1 a was observed in OPERA II $(p=0.004)$. Analyses of change in brain volume from week 24 to week 96, change in the 36-Item ShortForm Health Survey (SF-36) physical component summary (PCS) score from baseline to week 96, and the proportion of patients with no evidence of disease activity (NEDA; defined as no relapse, no 12-week or 24-week CDP, no new or enlarged $T_{2}$ lesions and no gadolinium-enhancing $T_{1}$ lesions) by week 96 favoured ocrelizumab (non-confirmatory nominal $p$-values $<0.05$ vs interferon $\beta$-1a in OPERA I and/or II) [10].

2.1.1.1 During Open-Label Extension After 2 years of double-blind treatment in the OPERA trials, the majority of patients ( $n=702$ and 623 initially randomized to ocrelizumab and interferon $\beta-1 \mathrm{a}$, respectively) entered an openlabel extension (OLE) during which all patients received ocrelizumab [18]. Three years of treatment in the OLE (total treatment duration 5 study years) was completed by $89 \%$ of patients who received continuous ocrelizumab (i.e. in both OPERA and OLE) and $88 \%$ of patients initially randomized to interferon $\beta-1 \mathrm{a}$ and switched to ocrelizumab in the OLE. 
Table 2 Efficacy of ocrelizumab in the management of relapsing multiple sclerosis: results of OPERA I and OPERA II [10]

ARR at week 12-week CDP ${ }^{\mathrm{a}} \quad$ 24-week $\mathrm{CDP}^{\mathrm{a}} \quad$ 12-week CDI ${ }^{\mathrm{b}} \quad$ Mean no. of lesions per MRI scan by week 96
96
(\% of pts)
(\% of pts)
(\% of pts)
$\mathrm{Gd}+$ on $\mathrm{T}_{1} \mathrm{~W} \quad \mathrm{NNEH}$ on $\mathrm{T}_{2} \mathrm{~W} \quad \mathrm{NH}$ on $\mathrm{T}_{1} \mathrm{~W}$

\begin{tabular}{|c|c|c|c|c|c|c|c|}
\hline \multicolumn{8}{|l|}{ OPERA I ${ }^{c}$} \\
\hline $\begin{array}{c}\text { Ocrelizumab } \\
(n=410)\end{array}$ & 0.16 & 7.6 & 5.9 & 20.0 & 0.02 & 0.32 & 0.42 \\
\hline $\begin{array}{c}\text { Interferon } \beta-1 \mathrm{a} \\
(n=411)\end{array}$ & 0.29 & 12.2 & 9.5 & 12.4 & 0.29 & 1.41 & 0.98 \\
\hline $\begin{array}{l}\mathrm{RR} / \mathrm{HR}(95 \% \mathrm{CI}) \text { or } \\
\text { difference }(\%)\end{array}$ & $\begin{array}{l}0.54 * * * \\
(0.40-0.72)\end{array}$ & $\begin{array}{l}0.57 \\
(0.37-0.90)\end{array}$ & $\begin{array}{l}0.57 \\
(0.34-0.95)\end{array}$ & 61 & $\begin{array}{l}0.06 * * * \\
(0.03-0.10)\end{array}$ & $\begin{array}{l}0.23 * * * \\
(0.17-0.30)\end{array}$ & $\begin{array}{l}0.43 * * * \\
(0.33-0.56)\end{array}$ \\
\hline \multicolumn{8}{|l|}{ OPERA II ${ }^{\mathrm{c}}$} \\
\hline $\begin{array}{c}\text { Ocrelizumab } \\
(n=417)\end{array}$ & 0.16 & 10.6 & 7.9 & 21.4 & 0.02 & 0.33 & 0.45 \\
\hline $\begin{array}{l}\text { Interferon } \beta-1 \mathrm{a} \\
(n=418)\end{array}$ & 0.29 & 15.1 & 11.5 & 18.8 & 0.42 & 1.90 & 1.26 \\
\hline $\begin{array}{l}\mathrm{RR} / \mathrm{HR}(95 \% \mathrm{CI}) \text { or } \\
\text { difference }(\%)\end{array}$ & $\begin{array}{l}0.53 * * * \\
(0.40-0.71)\end{array}$ & $\begin{array}{l}0.63 \\
(0.42-0.92)\end{array}$ & $\begin{array}{l}0.63 \\
(0.40-0.98)\end{array}$ & 14 & $\begin{array}{l}0.05 * * * \\
(0.03-0.09)\end{array}$ & $\begin{array}{l}0.17 * * * \\
(0.13-0.23)\end{array}$ & $\begin{array}{l}0.36^{* * * *} \\
(0.27-0.47)\end{array}$ \\
\hline \multicolumn{8}{|c|}{ Pooled OPERA I and II } \\
\hline $\begin{array}{c}\text { Ocrelizumab } \\
(n=827)\end{array}$ & NA & 9.1 & 6.9 & 20.7 & NA & NA & NA \\
\hline $\begin{array}{l}\text { Interferon } \beta-1 \mathrm{a} \\
(n=829)\end{array}$ & NA & 13.6 & 10.5 & 15.6 & NA & NA & NA \\
\hline $\begin{array}{r}\text { HR }(95 \% \mathrm{CI}) \text { or } \\
\text { difference }(\%)\end{array}$ & NA & $\begin{array}{l}0.60 * * * \\
(0.45-0.81)\end{array}$ & $\begin{array}{l}0.60 * * \\
(0.43-0.84)\end{array}$ & $33^{*}$ & NA & NA & NA \\
\hline
\end{tabular}

Primary endpoint (ARR) and MRI secondary endpoints were analysed in the ITT populations of individual trials; secondary endpoints of CDP and CDI were prespecifed to be analysed in the pooled OPERA I and II ITT populations (data from individual trials displayed for completeness) $A A R$ annualized relapse rate, $B L$ baseline, $C D I$ confirmed disability improvement, $C D P$ confirmed disability progression, EDSS Expanded Disability Status Scale, $G d+$ gadolinium-enhancing, $H R$ hazard ratio, $M R I$ magnetic resonance imaging, $N A$ not assessed within hierarchical testing procedure, $N H$ new hypointense, $N N E H$ new or enlarged hyperintense, $p t s$ patients, $R R$ rate ratio, $T_{1 / 2} W \mathrm{~T}_{1}$ - or $\mathrm{T}_{2}$-weighted MRI scan

${ }^{*} p=0.02, * * p=0.003$, *** $p<0.001 \mathrm{vs}$ interferon $\beta$-1a (displayed for hierarchically assessed primary and secondary endpoints only)

${ }^{a}$ Disability progression defined as a $\geq 1$.0-point increase from BL in EDSS score (or $\geq 0.5$-point increase if BL EDSS score $>5.5$ ) sustained for $\geq 12$ (12-week CDP) or $\geq 24$ weeks (24-week CDP) through week 96

${ }^{\mathrm{b}}$ Assessed in pts with BL EDSS scores $\geq 2.0$; disability improvement defined as a $\geq 1.0$-point reduction from BL EDSS score (or $\geq 0.5$ point if BL EDSS score $>5.5$ ) sustained for $\geq 12$ weeks through week 96

${ }^{c}$ See text for dosage and regimen details

Through these years, a near complete suppression of MRI disease activity was seen in patients receiving continuous ocrelizumab treatment. In these patients, the unadjusted rate of total gadolinium-enhancing $\mathrm{T}_{1}$ lesions was 0.006 at year 5 of treatment (vs 0.017 at year 2) and that of new or newly enlarged $\mathrm{T}_{2}$ lesions was 0.031 over year 5 (vs 0.063 over year 2). In patients switched from interferon $\beta$-1a to ocrelizumab in the OLE, the respective rates were 0.004 (vs 0.491 ) and 0.038 (vs 2.583). While there were no betweengroup differences in MRI lesion counts at year 5, patients continuously treated with ocrelizumab had less brain atrophy than those switched from interferon $\beta$-1a (as measured by adjusted rates of change in whole brain volume, cortical grey matter volume and white matter volume from doubleblind baseline; $p<0.01$ for all) [18].

The therapeutic benefits of ocrelizumab were maintained over 7.5 study years of follow-up (5.5 years in the OLE, completed by $76 \%$ of patients who entered the OLE) [19]. At OLE year 5.5, the adjusted ARR was 0.03 in patients who received ocrelizumab during both double-blind treatment and the OLE (vs 0.12 pre-switch) and 0.03 in patients who were switched from interferon $\beta$-1a to ocrelizumab on entering the OLE (vs 0.20 pre-switch). Rates of 48-week CDP were $17.9 \%$ and $21.5 \%$ in the respective groups (compared with $4.1 \%$ and $8.5 \%$ at the end of double-blind treatment), while rates of requiring a walking aid (i.e. EDSS $\geq 6.0$ ) were $6.6 \%$ and $9.5 \%$ (compared with $0.8 \%$ and $3.1 \%$ ). Over the double-blind period and OLE, patients who continuously received ocrelizumab had a $23 \%$ lower risk of 48 -week CDP (HR 0.77; 95\% CI 0.60-0.98; $p=0.034$ ) and 35\% lower risk of requiring a walking aid (HR 0.65 ; 95\% CI 0.44-0.97; $p=0.034$ ) than patients who switched from interferon $\beta$-1a to ocrelizumab [19]. 


\subsubsection{In Active RMS}

PRO-MSACTIVE enrolled patients with active RMS based on clinical and/or imaging features [11]. Patients received ocrelizumab $600 \mathrm{mg}$ infusions every 24 weeks (initial dose administered as two $300 \mathrm{mg}$ infusions 14 days apart) for 48 weeks ( $n=422$ enrolled). The primary endpoint was the percentage of patients free of disease activity (including protocol-defined relapse since enrolment, $\mathrm{T}_{1}$ gadoliniumenhancing lesions and new or enlarging $\mathrm{T}_{2}$ lesions) at week 48. Interim data from patients who have completed 48 weeks of treatment thus far $(n=335)$ are available. At baseline, most patients $(91 \%)$ included in the interim analysis had a diagnosis of RRMS [11].

Of the ocrelizumab recipients in the interim analysis, $65.1 \%$ (95\% CI 59.7-70.2) were free of all disease activity events at week 48 , with $87.2 \%$ having no protocol-defined relapse, $83.6 \%$ having no gadolinium-enhancing $\mathrm{T}_{1}$ lesions and $76.1 \%$ having no new or enlarging $\mathrm{T}_{2}$ lesions [11]. Without MRI re-baselining, there was no evidence of MRI activity in $69.0 \%$ of patients. At week $48,16.4 \%$ of patients had gadolinium-enhancing $\mathrm{T}_{1}$ lesions (vs $52.1 \%$ at baseline) and $23.9 \%$ had new or enlarging $\mathrm{T}_{2}$ lesions (vs $93.9 \%$ at baseline). The adjusted ARR was 0.133 (95\% CI 0.072-0.246). The mean EDSS score was 2.56 at week 48 (vs 2.81 at baseline), with scores remaining stable in $66.9 \%$ of patients and improving in $18.5 \%$ [11].

\subsubsection{In Patients with Suboptimal Response to Prior DMTs}

CASTING [15] and CHORDS [14] enrolled patients aged 18-55 years with a diagnosis of RRMS (based on the 2010 revised McDonald criteria), a disease duration from first symptom of $<10$ years [15] or $\leq 12$ years [14] and a screening EDSS score of 0.0-4.0 [15] or 0.0-5.5 [14]. Eligible patients had received $\leq 2$ [15] or $\leq 3$ [14] other DMTs prior to screening and had discontinued the most recent adequately used DMT due to suboptimal disease control $[14,15]$. In both trials, patients received ocrelizumab $600 \mathrm{mg}$ (administered as two $300 \mathrm{mg}$ infusions on days 1 and 15 for the initial dose and a single $600 \mathrm{mg}$ infusion thereafter) every 24 weeks for 96 weeks ( $n=680$ evaluated in CASTING and 576 in CHORDS). The primary endpoint was NEDA (with prespecified MRI re-baselining at week 8 [15]), defined as the absence of 24-week CDP, protocoldefined relapse, gadolinium-enhancing $\mathrm{T}_{1}$ lesions and new or enlarging $\mathrm{T}_{2}$ lesions, over 96 weeks $[14,15]$.

The proportion of ocrelizumab recipients with NEDA over 96 weeks was $48.1 \%$ in CHORDS [14] and 74.8\% (with MRI re-baselining at week 8) in CASTING [15]. Most patients were free from 24-week CDP $(89.6 \%$ in CHORDS and $87.5 \%$ in CASTING), protocol-defined relapses $(89.6 \%$ and $89.8 \%$ ), gadolinium-enhancing $\mathrm{T}_{1}$ lesions $(95.5 \%$ and
97.7\%) and new or enlarging $\mathrm{T}_{2}$ lesions (59.5\% and 91.5\%) $[14,15]$. In CHORDS, there was no significant difference between subgroups based on previous DMT use (1 vs $>1$ DMT) in the proportion of patients with NEDA over 96 weeks [50.9\% (163/320) vs 44.5\% (114/256)] [14]. The adjusted ARRs were 0.046 in CHORDS and 0.03 in CASTING [14, 15].

Ocrelizumab was efficacious with respect to MRI measures of inflammation and disease progression, based on data from CHORDS [14]. The proportion of patients with $T_{1}$ gadolinium-enhancing lesions reduced from $3.3 \%$ at week 24 to $1.1 \%$ at week 96 and the proportion with new and/or enlarging $\mathrm{T}_{2}$ lesions reduced from 36.0 to $2.7 \%$. The mean change in overall $\mathrm{T}_{2}$ lesion volume from baseline to week 96 was $-0.56 \mathrm{~cm}^{3}$ (vs $-0.48 \mathrm{~cm}^{3}$ at week 24) [14].

Following the 96-week treatment period in CASTING, eligible patients rolled over to LIBERTO, an ongoing openlabel extension study ( $n=439$, as of October 2020) [20]. Patients in LIBERTO continued to receive ocrelizumab 600 mg every 24 weeks. Based on 1-year interim results, NEDA was achieved in $82.5 \%$ (235/285) of patients from the second year to the third year of treatment. Over the 3-year treatment period from CASTING baseline to LIBERTO week 48, $59.4 \%$ (190/320) of patients had NEDA, 68.1\% had freedom from CDP or relapse and $86.6 \%$ had no MRI activity [20].

\subsection{In Primary Progressive Multiple Sclerosis}

ORATORIO enrolled adults aged 18-55 years with PPMS (based on the 2005 revised McDonald criteria), an EDSS score of 3.0-6.5 at screening, a Functional Systems Scale pyramidal functions component score of $\geq 2$, an elevated IgG index or at least one $\operatorname{IgG}$ oligoclonal band detected in the cerebrospinal fluid (or documented history thereof) and MS symptoms for $<15$ years (in patients with an EDSS score of $>5$ ) or $<10$ years (in patients with an EDSS score of $\leq 5$ ) at screening [16]. Patients received ocrelizumab 600 $\mathrm{mg}$ (administered as two $300 \mathrm{mg}$ infusions given 2 weeks apart) or matching placebo every 24 weeks, with randomization in a 2:1 ratio and stratified by geographical region and age. Double-blind treatment continued for a minimum of five doses (120 weeks) and until the occurrence of $\approx 253$ events of 12-week CDP. The primary endpoint was the percentage of patients with 12-week CDP. At baseline, the mean time since diagnosis of PPMS was $\approx 3$ years in each treatment group. Most patients (88\%) had not used a DMT in the 2 years prior to trial entry [16].

Ocrelizumab was effective in delaying clinical progression in patients with PPMS, significantly reducing the proportion of patients with 12-week CDP relative to placebo (Table 3; primary endpoint) [16]. The relative risk reduction with ocrelizumab versus placebo was $24 \%$ (HR 0.76; $95 \%$ CI $0.59-0.98 ; p=0.03$ ) [16]. While ORATORIO was not 
powered to demonstrate between-group differences among subgroups [16], prespecified subgroup analyses of 12-week CDP suggested somewhat more pronounced treatment benefits in patients with baseline $\mathrm{T}_{1}$ gadolinium-enhancing lesions (HR 0.65) than those without (HR 0.84) and in younger patients (i.e. $\leq 45$ years; HR 0.64 ) than in older patients (i.e. $>45$ years; HR 0.88) [8]. Other exploratory subgroup analyses indicated that $\approx 36 \%$ of female patients had 12-week CDP irrespective of treatment arm, while the 12 -week CDP rates were $\approx 30 \%$ and $43 \%$ in male patients receiving ocrelizumab and placebo [7].

Other measures of clinical or MRI progression were also improved with ocrelizumab versus placebo [16]. Ocrelizumab significantly reduced both the proportion of patients with 24-week CDP (a relative risk reduction of 25\%) and the change from baseline to week 120 in timed 25-foot walk (T25FW) performance (Table 3). In terms of MRI outcomes, ocrelizumab significantly improved mean changes in total volume of $\mathrm{T}_{2}$ hyperintense lesions from baseline to week 120 and in brain volume from week 24 to 120 . Ocrelizumab and placebo recipients did not significantly differ with respect to change from baseline to week 120 in physical health-related quality of life, as assessed by SF-36 PCS score (Table 3) [16].

Results of prespecified exploratory analyses gave further support to the benefits of ocrelizumab [16]. Analyses of 12-week and 24-week composite CDP [defined as the first confirmed occurrence of increase in EDSS score, $\geq 20 \%$ increase in T25FW time, or $\geq 20 \%$ increase in 9-hole peg test (9HPT) completion time] favoured ocrelizumab over placebo (HRs 0.74 and 0.71; both $p \leq 0.001$ ), as did the adjusted mean number of new or enlarging $\mathrm{T}_{2}$ hyperintense lesions from baseline to week 120 ( 0.31 vs $3.88 ; p<0.001$ )
[16]. Ocrelizumab was protective against the progression of upper extremity impairment, reducing the risk of 12-week or 24-week 9HPT progression relative to placebo (HRs 0.56 and 0.55 ; both $p<0.001)[16,21]$.

\subsubsection{During Open-Label Extension}

The majority (97\%) of patients who completed the doubleblind treatment period (to week 144) of ORATORIO entered the OLE phase, during which all patients received ocrelizumab [22]. This OLE is currently ongoing (planned completion date December 2022). Interim efficacy data are available $(n=451$ ongoing and followed for $\geq 6.5$ study years, including 3.5 study years in the OLE; one study year is equal to 48 weeks). All analyses were post hoc [22].

The benefits of ocrelizumab for disability progression were sustained over $\geq 6.5$ years of treatment in the interim analysis [22]. Compared with patients who had initially received placebo, patients continuously treated with ocrelizumab had lower rates of progression on most measures of 24-week CDP, which included EDSS (progression rate $51.7 \%$ vs $64.8 \% ; p=0.0018)$, 9HPT completion time (30.6\% vs $43.1 \% ; p=0.0035)$, T25FW performance $(63.2 \%$ vs $70.7 \% ; p=0.058)$ and composite progression $(73.2 \%$ vs $83.3 \% ; p=0.0023$ ), from the double-blind baseline to week 312 . They also had a lower rate of progression to requiring a wheelchair (EDSS $\geq 7 ; 11.5 \%$ vs $18.9 \% ; p=0.0274)$; across the full study, the overall risk of progression to requiring a wheelchair was $42 \%$ lower with continuous ocrelizumab therapy (HR 0.58; 95\% CI 0.38-0.89; $p=0.0112$ ) [22]. Based on extrapolations of data from ORATORIO (doubleblind period and OLE; 312 weeks total follow-up), ocrelizumab was predicted to delay the time to 24 -week confirmed

Table 3 Efficacy of ocrelizumab in the management of primary progressive multiple sclerosis: results of ORATORIO [16]

\begin{tabular}{|c|c|c|c|c|c|c|}
\hline & $\begin{array}{l}12-\text { week CDP } \\
\text { (\% of pts) }\end{array}$ & $\begin{array}{l}\text { 24-week CDP } \\
\text { (\% of pts) }\end{array}$ & $\begin{array}{l}\mathrm{T} 25 \mathrm{FW} \text { performance } \\
\left(\% \Delta^{\mathrm{b}} \text { from BL to }\right. \\
\text { week } 120)\end{array}$ & $\begin{array}{l}\mathrm{T}_{2} \mathrm{~W} \text { lesion volume } \\
\left(\% \Delta^{\mathrm{b}} \text { from BL to week }\right. \\
120)\end{array}$ & $\begin{array}{l}\text { Brain volume } \\
\left(\% \Delta^{\mathrm{b}} \text { from week }\right. \\
24 \text { to } 120)\end{array}$ & $\begin{array}{l}\text { SF-36 PCS score } \\
\left(\Delta^{\mathrm{b}} \text { from BL to }\right. \\
\text { week 120) }\end{array}$ \\
\hline $\begin{array}{l}\text { Ocrelizumab }^{c} \\
(n=488)\end{array}$ & 32.9 & 29.6 & 38.9 & -3.37 & -0.90 & -0.7 \\
\hline $\operatorname{Placebo}^{c}(n=244)$ & 39.3 & 35.7 & 55.1 & 7.43 & -1.09 & -1.1 \\
\hline $\begin{array}{l}\text { HR or relative } \\
\text { difference } \\
(95 \% \mathrm{CI})\end{array}$ & $\begin{array}{l}0.76^{*}(0.59 \text { to } \\
0.98)\end{array}$ & $\begin{array}{l}0.75^{*}(0.58 \text { to } \\
0.98)\end{array}$ & $\begin{array}{l}29.3^{*}(-1.6 \text { to } \\
51.5)\end{array}$ & $\begin{array}{l}0.90 * * \text { ( } 0.88 \text { to } \\
0.92)\end{array}$ & $\begin{array}{l}17.5 * \text { (3.2 to } \\
29.3)\end{array}$ & $\begin{array}{l}0.38(-1.05 \text { to } \\
1.80)\end{array}$ \\
\hline
\end{tabular}

Primary (12-week CDP) and secondary endpoints, analysed in the intention-to-treat population and assessed hierarchically in order displayed $B L$ baseline, $C D P$ confirmed disability progression, $H R$ hazard ratio, pts patients, SF-36 PCS 36-Item Short-Form Health Survey Physical Component Summary, $T 25 F W$ timed 25 -foot walk, $T_{2} W \mathrm{~T}_{2}$-weighted MRI scan, $\Delta$ change

$* p<0.05, * * p<0.001$ vs placebo

${ }^{a}$ Disability progression defined as a $\geq 1.0$-point increase from BL in EDSS score (or $\geq 0.5$-point increase if BL score $>5.5$ ) sustained for $\geq 12$ weeks (12-week CDP) or $\geq 24$ weeks (24-week CDP)

${ }^{\mathrm{b}}$ Mean percent $\Delta$ (T25FW; brain volume), adjusted geometric mean percent $\Delta$ (total $\mathrm{T}_{2}$ lesion volume) or adjusted mean $\Delta$ (SF-36 PCS score)

${ }^{\mathrm{c}} \mathrm{See}$ text for dosage and regimen details 
wheelchair requirement (i.e. EDSS $\geq 7.0)$ by 7.1 years $(95 \%$ CI -4.3 to 18.4) relative to placebo [23]. The extrapolated median time to 24-week confirmed EDSS $\geq 7.0$ was 19.2 years with ocrelizumab versus 12.1 years with placebo. The plausibility of these extrapolations is supported by observed data from an independent real-world PPMS cohort (MSBase registry), in which the median time to 24-week confirmed EDSS $\geq 7.0$ was 12.4 years [23].

More recent disability progression results from after 8 years of treatment (week 408 data) continued to favour continuous ocrelizumab treatment; relative to patients who initially received placebo, patients continuously treated with ocrelizumab had reduced $(p \leq 0.002)$ risks of first 48 -week CDP based on EDSS or 9HPT ( $p=0.057$ for reduction in risk of first 48-week EDSS $\geq 7$ ) [24].

With respect to MRI outcomes, suppression of lesion activity was sustained (and near complete) over long-term treatment with ocrelizumab [22]. Patients who received continuous ocrelizumab had smaller increases from double-blind baseline in $\mathrm{T}_{2}$ lesion volume $(0.45 \%$ vs $13.00 \%$ in patients who switched from placebo to ocrelizumab; $p<0.0001)$ and $\mathrm{T}_{1}$ hypointense lesion volume (36.68\% vs $60.93 \% ; p=0.0008)$ at OLE week 144 [22].

\subsection{In Real-World Studies}

The effectiveness of ocrelizumab in the treatment of patients with MS in a real-world clinical setting has been investigated in several large studies $(n>300)$ [25-32], the early results of which appear to confirm the therapeutic benefits demonstrated in clinical trials.

An interim analysis of the effectiveness of ocrelizumab in the treatment of patients with RMS and PPMS is available from the non-interventional CONFIDENCE study [27]. Over one year of observation, $83.6 \%$ of ocrelizumab recipients with RMS and $93.2 \%$ with PPMS experienced treatment success (defined as no relapse, progression or treatment discontinuation due to an adverse event); mean changes in EDSS from baseline to after one year of treatment were 0.0 and 0.1 in the respective groups. Most ocrelizumab recipients with RMS (85.3\%) experienced no relapses. CONFIDENCE aims to collect $\leq 10$ years of data from newly treated ocrelizumab recipients $(n=3000)$ and recipients of other select DMTs $(n=1500)$ in $\approx 250$ German centres. The interim analysis included data from $\approx 559$ ocrelizumab recipients $(\approx 82 \%$ with RMS and $\approx 18 \%$ with PPMS $)$ with one year of follow-up [27].

Early analyses of data from the international MSBase Registry [28] and Danish Multiple Sclerosis Registry [30] also indicate that ocrelizumab is an effective treatment for MS. In MSOCR-R, a prospective MSBase cohort study of patients with RRMS newly treated with ocrelizumab $(n=$ 800 eligible as of April 2021), most ocrelizumab recipients
(91\%) remained free from relapse after 2 years [28]. The ARR was 0.073. Kaplan-Meier estimates of 6-month CDPfree survival were $96 \%$ at 12 months and $91 \%$ at 24 months, while estimates of 6-month CDI were $7 \%$ and $10 \%$. Patients had a median exposure to ocrelizumab of 1.8 years [28]. In an observational cohort study with prospectively enrolled cases ( $n=1104$ initiating treatment with ocrelizumab; 946, 97 and 61 with RRMS, SPMS and PPMS, respectively) using data from the nationwide population-based Danish Multiple Sclerosis Registry, 9.3\% of the total cohort experienced relapses during ocrelizumab treatment (median time to first relapse 4.2 months) [30]. The mean ARR was 0.09 after initiation of ocrelizumab versus 0.58 in the year prior to initiation. The overall rate of 24-week CDP at month 12 was $8.4 \%(7.2 \%, 10.4 \%$ and $20.5 \%$ in patients with RRMS, SPMS and PPMS, respectively). Most patients (94.5\%) were free of MRI disease activity after $\approx 1$ year of treatment. The median duration of follow-up was 16 months [30].

Data on real-world outcomes with ocrelizumab versus other DMTs are available from an analysis using a US commercial claims database [29]. This analysis included data from patients with MS enrolled for $\geq 1$ year pre- and postinitiation of a new DMT $(n=729,662,1109$ and 213 initiating ocrelizumab, comparator injectable DMTs, comparator oral DMTs or natalizumab). The pre-initiation year ARR was 0.54 in the ocrelizumab group ( 0.60 in the quarter prior to initiation), 0.33 in the injectable DMT group (0.85), 0.33 in the oral DMT group (0.75) and 0.60 in the natalizumab group (1.1). From the pre-initiation year to the post-initiation year, the changes in ARR were - 0.31 with ocrelizumab, -0.08 with injectable DMTs, -0.12 with oral DMTs and -0.19 with natalizumab [29].

\section{Tolerability of Ocrelizumab}

Ocrelizumab $600 \mathrm{mg}$ administered intravenously every 24 weeks was generally well tolerated in patients with RMS and PPMS in clinical trials (e.g. [10, 11, 14-16, 33, $34])$ and in a real-world setting (e.g. [30, 35, 36]). In the pooled OPERA I and II safety populations, the proportion of patients reporting adverse events (AEs) was the same with ocrelizumab as with interferon $\beta-1$ a (83.3\%) [10]. Serious AEs occurred in $6.9 \%$ of ocrelizumab recipients (vs $8.7 \%$ of interferon $\beta$-1a recipients) and AEs led to discontinuation in $3.5 \%$ (vs $6.2 \%$ ) [10]. In the ORATORIO safety population, AEs occurred in $95.1 \%$ of ocrelizumab recipients (vs $90.0 \%$ of placebo recipients), were serious in $20.4 \%$ (vs $22.2 \%$ ) and led to discontinuation of the trial agent in $4.1 \%$ (vs 3.3\%) [16]. No new safety signals were identified over $\leq 7$ years of continuous treatment with ocrelizumab during the clinical trials and OLEs [37]. 
During the pivotal clinical trials, infusion-related reactions (IRRs) were the most frequently reported AEs in ocrelizumab recipients (Fig. 1) [10, 16]. Common IRRs included pruritus, rash, flushing and throat irritation [38]. IRRs were predominantly mild to moderate in severity and most frequently observed with the first infusion, decreasing in frequency thereafter. Severe IRRs occurred in $2.4 \%$ of ocrelizumab recipients in the OPERA trials (vs $0.1 \%$ of interferon $\beta$-1a recipients) and $1.2 \%$ in ORATORIO (vs $1.7 \%$ of placebo recipients). There was one life-threatening case of bronchospasm with ocrelizumab (in OPERA I; patient discontinued ocrelizumab and event resolved on same day without sequelae) and no fatal IRRs. Patients were premedicated with methylprednisolone before ocrelizumab infusions and had the option to receive additional prophylaxis, which reduced the incidence of IRRs. IRRs that did occur were managed effectively through infusion rate adjustments and the treatment of symptoms [38]. Premedication with intravenous methylprednisolone $100 \mathrm{mg}$ (or equivalent) and an antihistamine should be administered prior to every ocrelizumab infusion [7, 8].

The administration of ocrelizumab $600 \mathrm{mg}$ infusions over a shorter duration ( $2 \mathrm{~h}$; Sect. 4) did not substantially alter the safety profile of ocrelizumab relative to infusions over the conventional duration $(3.5 \mathrm{~h})$ in the randomized, doubleblind ENSEMBLE PLUS substudy in patients with RRMS ( $n=373$ and 372 in the conventional and shorter duration groups) [33]. During or after the first randomized dose, IRRs occurred in $28.8 \%$ of patients in the shorter infusion group and $26.5 \%$ of patients in the conventional infusion group (difference $2.44 \%$; $95 \%$ CI -3.83 to 8.71 ) [primary endpoint; Fig. 1c]. The severity of IRRs and proportion of patients experiencing IRRs requiring symptomatic treatment (e.g. antihistamines, antiemetics, analgesics) were generally comparable between groups. IRRs requiring intervention (i.e. infusion slowing or temporary interruption) were somewhat more frequent with the shorter infusions (Fig. 1c). In each group, the majority of IRRs were mild or moderate in severity and resolved without sequelae; no IRRs led to treatment discontinuation and there were no serious, lifethreatening or fatal IRRs in either group [33]. The results of ENSEMBLE PLUS were supported by those of the CHORDS extension substudy ( $n=129$ with RRMS) [39] and the US open-label, phase IIIb SaROD shorter infusion study, which included several patients with PPMS (representing $9.5 \%$ of the 95 patients with MS in the shorter 600 mg infusion cohort) [34].

Infections such as upper respiratory tract infection, nasopharyngitis and urinary tract infection were common in ocrelizumab recipients during the pivotal clinical trials (Fig. 1) and were mostly of mild or moderate severity [10, 16]. Across several clinical trials and OLE periods up to January 2020 [ $n=5680$ ocrelizumab recipients with MS;
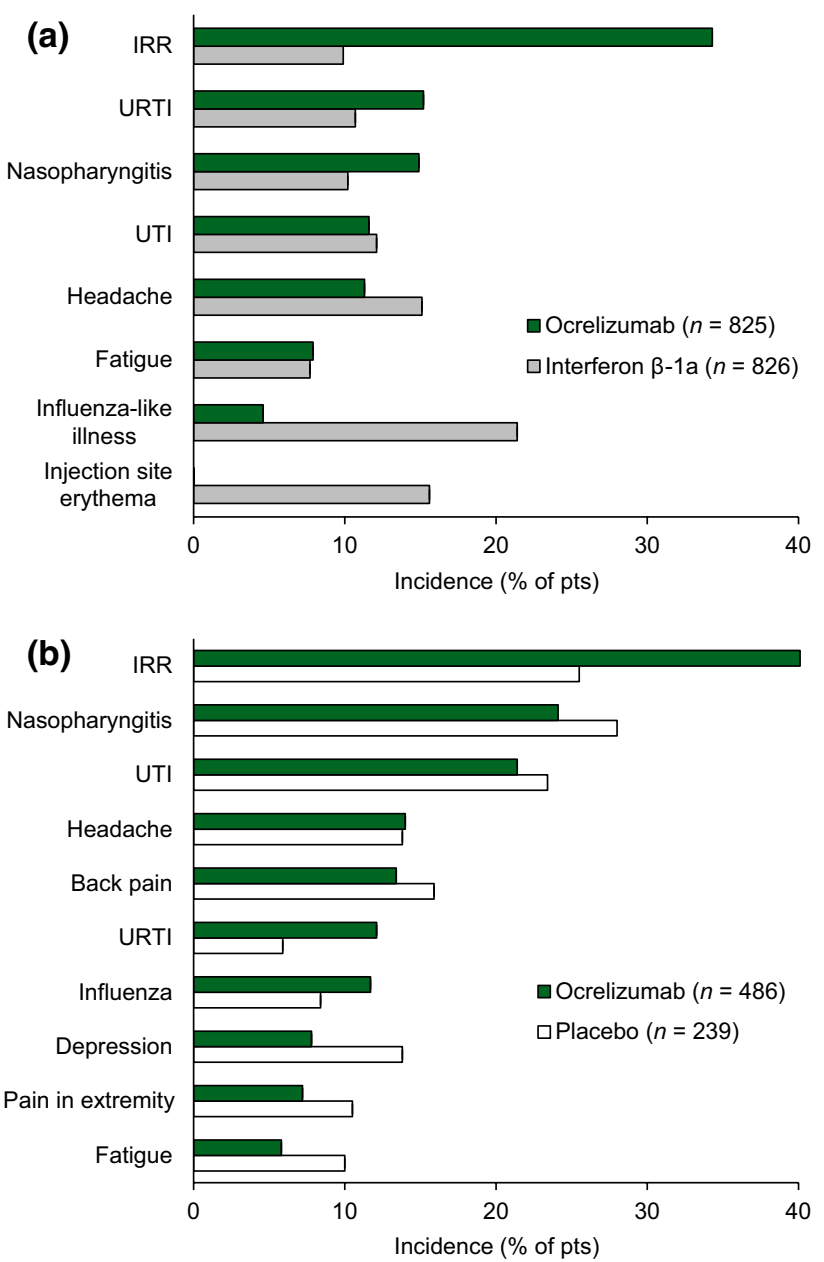

(c)

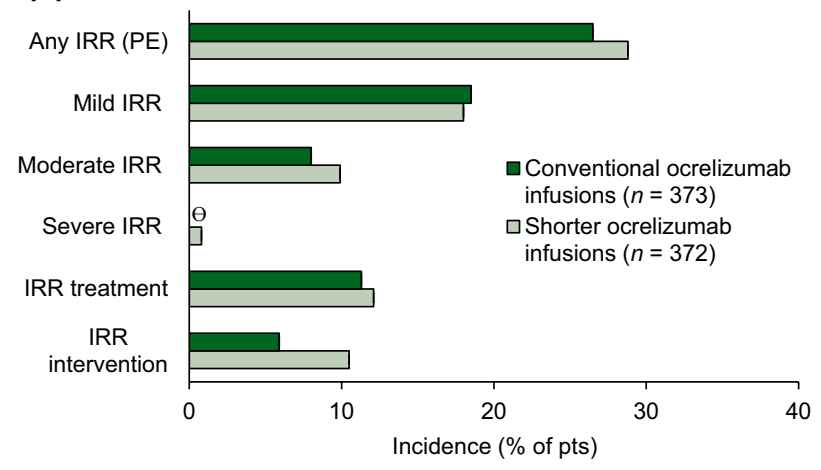

Fig. 1. Adverse events ( $\geq 10 \%$ incidence in any treatment arm) occurring with ocrelizumab in patients with a relapsing multiple sclerosis (pooled OPERA-I and II safety populations [10]) and b primary progressive multiple sclerosis (ORATORIO [16]), and c IRRs occurring with ocrelizumab in patients with relapsing-remitting multiple sclerosis (ENSEMBLE-PLUS [33]; first randomized infusion). IRR infusion-related reaction, $P E$ primary endpoint, $U R T I$ upper respiratory tract infection, $U T I$ urinary tract infection, $\theta$ zero incidence

18,218 patient-years (PY) of exposure], infections were reported at a rate of 76.2 events/100 PY and serious infections were reported at a rate of 2.01 events/100 PY [37]. This 
serious infection rate is consistent with the range reported in epidemiological data [37]. Serious opportunistic infections were rare during the OPERA and ORATORIO trials (three potential cases reported across the two OLEs) and there were no cases of progressive multifocal leukoencephalopathy (PML) [18, 22]. As of 31 July 2020, an estimated 174,508 patients with MS have been treated with ocrelizumab globally, including 167,684 patients who initiated ocrelizumab during post-marketing experience (resulting in an estimated 249,971 PY of exposure) [37]. There have been nine cases of confirmed PML in ocrelizumab recipients during postmarketing experience, eight of which occurred in patients previously treated with DMTs $[22,37]$. Ocrelizumab is contraindicated in patients with current active infections or in a severely immunocompromised state in the EU [8] and in patients with active hepatitis B virus infection in the USA [7].

Based on data from ongoing clinical trials, post-marketing use and an external health record database, coronavirus disease 2019 (COVID-19) cases in ocrelizumab recipients were predominantly of mild to moderate severity, consistent with those in the general population and patients with MS [40]. There was no association between duration of ocrelizumab exposure and COVID-19 rates. The risk factors for severe COVID-19 outcomes in ocrelizumab recipients with MS were the same as those in the general population (e.g. older age, comorbidities). Rates of hospitalization, invasive ventilation and death in ocrelizumab recipients with MS were similar to those in non-ocrelizumab treated patients with MS [40]. Although antibody responses to vaccines are attenuated in ocrelizumab recipients (Table 1), vaccination against COVID-19 (with non-live vaccines) may still be expected to offer some protection [40-42]. For non-live vaccines requiring two doses (including COVID-19 vaccines), the first dose should be administered $\approx 12$ weeks after the most recent ocrelizumab infusion and the second dose given no less than 4 weeks before the next infusion [37].

Ocrelizumab may increase the risk of malignancies (e.g. breast cancer) [8]. Across ocrelizumab clinical trials and OLE periods up to January 2020, malignancies were reported at a rate of 0.46 events/100 PY [37]. Although this rate is consistent with the range reported in epidemiological data [37], the use of ocrelizumab is nevertheless contraindicated in patients with known active malignancies in the EU [8].

As with other therapeutic proteins, there is the potential for immunogenicity with ocrelizumab [7]. Of patients treated with ocrelizumab in the pivotal trials $(n=1311), 12$ $(0.9 \%)$ tested positive for antidrug antibodies (two of whom tested positive for neutralizing antibodies) $[7,8]$. This is consistent with the humanized nature of ocrelizumab. The impact of antidrug antibodies on the safety and efficacy of ocrelizumab is unknown $[7,8]$.

\section{Dosage and Administration of Ocrelizumab}

In the EU, ocrelizumab is indicated for the treatment of adults with relapsing forms of MS with active disease (as defined by clinical or imaging features), or early PPMS (in terms of disease duration and disability level) with imaging features characteristic of inflammatory activity [8]. In the USA, ocrelizumab is indicated for the treatment of adults with relapsing forms of MS (including clinically isolated syndrome, RRMS and active SPMS) or PPMS [7]. The recommended dosage is $600 \mathrm{mg}$ every 6 months, administered intravenously as two $300 \mathrm{mg}$ infusions 2 weeks apart for the first dose and single $600 \mathrm{mg}$ infusions thereafter $[7,8]$. The initial two infusions should each be given over $\approx 2.5 \mathrm{~h}$ in the EU ( $\geq 2.5 \mathrm{~h}$ in USA [7]) [8]. Subsequent doses can be given over $\approx 3.5 \mathrm{~h}(\geq 3.5 \mathrm{~h}$ in the USA [7]) or, if no serious IRR has occurred with any previous ocrelizumab infusion, $\approx 2 \mathrm{~h}$ ( $\geq 2 \mathrm{~h} \mathrm{[7])} \mathrm{[8].} \mathrm{Consult} \mathrm{local} \mathrm{prescribing} \mathrm{information} \mathrm{for}$ details concerning delayed or missed doses, premedication for IRRs, management of IRRs, contraindications, special warnings and precautions, and use in special populations.

\section{Place of Ocrelizumab in the Management of Multiple Sclerosis}

A variety of intravenously infused, injectable and oral DMTs are now approved for the treatment of relapsing forms of MS [4]. Ocrelizumab, the first anti-CD20 mAb to receive approval, selectively depletes CD20-expressing B cells while largely preserving normal immune function (Table 1) [9]. PPMS has proven more difficult to treat than RMS and ocrelizumab is currently the only DMT approved in this phenotype [4]. The 2018 ECTRIMS/EAN guidelines recommend that in patients with active RRMS, choice of treatment from among the wide range of available modestly effective to highly effective drugs (with ocrelizumab among these) should be reached in consultation with the individual patient and will depend on factors such as patient characteristics and comorbidities, disease severity/activity, drug safety profiles and drug accessibility (consensus statement) [3]. In patients with PPMS, ocrelizumab should be considered (weak recommendation) [3]. AAN guidelines similarly recommend that clinicians offer DMTs to patients with relapsing forms of MS and recent clinical relapses or MRI activity, with drug selection respecting patient preferences and incorporating relevant variables (e.g. administration route, patient lifestyle, cost, efficacy, safety/tolerability) [43]. Ocrelizumab should be offered to patients with PPMS who are likely to benefit from this therapy, unless treatment risks outweigh the benefits [43]. 
In the pivotal OPERA trials, ocrelizumab was significantly more effective than interferon $\beta$ - $1 \mathrm{a}$ in reducing relapse rates, as well as clinical and MRI measures of disease activity and progression, in patients with RMS (Sect. 2.1.1). OPERA results are supported by those from open-label phase IIIb and IV trials (including in patients with an inadequate response to previous DMTs; Sect. 2.1.3). Unlike other DMTs to date, ocrelizumab also demonstrated efficacy in the treatment of PPMS, significantly slowing disease progression relative to placebo in the pivotal ORATORIO trial (Sect. 2.2). The ORATORIO-HAND study is further investigating the effects of ocrelizumab on upper limb function in patients with PPMS [44]. Of importance for a chronic condition, the clinical benefits of ocrelizumab were sustained over long-term treatment ( $\geq 7.5$ study years in the pivotal trials and their OLEs), with partial suppression of disease activity and low rates of disability accrual (Sect. 2.1.1.1; 2.2.1). A number of large real-world studies of ocrelizumab in patients with RMS and PPMS are now underway and early results are consistent with the efficacy observed in clinical trials (Sect. 2.3)

Ocrelizumab was generally well tolerated in the treatment of patients with RMS and PPMS in clinical trials (including over $\leq 7$ years of continuous administration) and real-world experience (Sect. 3). The most common AEs in ocrelizumab recipients were IRRs and infections, which were primarily mild to moderate in severity. Importantly, in a pooled analysis of data from clinical trials and OLEs (representing $>18,000$ PY of exposure), serious infection and malignancy rates in ocrelizumab recipients were within the ranges reported in epidemiological data (Sect. 3). It should be noted that clinical trials of ocrelizumab have largely excluded older patients (i.e. $>55$ years of age; Sect. 2), who may be at greater risk of certain rare AEs. While no new safety signals have emerged during post-marketing experience amounting to $\approx 250,000 \mathrm{PY}$ of ocrelizumab exposure (Sect. 3), further long-term safety data from heterogeneous populations in real-world clinical practice (being collected in the ongoing CONFIDENCE, MANUSCRIPT and VERISMO safety studies, which will continue for $\leq 10$ years) are nevertheless awaited with interest.

In the absence of head-to-head comparisons between ocrelizumab and other approved treatments for relapsing forms of MS (aside from interferon $\beta$-1a; Sect. 2.1.1), recent network meta-analyses generally indicate that ocrelizumab is superior or comparable to other approved DMTs in efficacy while being similar with respect to safety [45-48]. Direct comparisons in clinical trials would be of use in ascertaining the relative position of ocrelizumab in the management of relapsing forms of MS.

With respect to patient convenience, ocrelizumab is infrequently administered (once every 6 months; Sect. 4) and is minimally disruptive to the personal and professional lives of patients [49]. Real-world data suggests that treatment adherence rates may be higher and discontinuation rates lower with ocrelizumab than with other intravenous, injectable or oral DMTs [50]. Of particular relevance in a pandemic setting (where access to infusion locations and individuals qualified in the treatment of chronic disease may be limited), ocrelizumab can now be administered as a rapid infusion (Sect. 4) without the safety profile being altered (albeit with slowing or interrupting of the infusion required in a minority of patients; Sect. 3). Shorter infusion times have the potential to reduce resource utilization and may be preferred by patients [51].

While modern DMTs improve outcomes in patients with MS, they do so at significant cost [52]. In a US cost-effectiveness analysis, ocrelizumab was the only approved DMT to approach the threshold for cost-effectiveness in the first-line treatment of RRMS but was not considered to be cost-effective in the treatment of PPMS [52]. In the UK, NICE recommends ocrelizumab as an option for the treatment of RRMS in adults with active disease if alemtuzumab is not suitable and if the ocrelizumab is provided at an agreed discounted price [49]. NICE also recommends ocrelizumab as an option for the treatment of early PPMS with imaging features characteristic of inflammatory activity in adults, considering cost-effectiveness estimates to be acceptable at the agreed price [53].

Based on rapidly accumulating evidence from clinical trials and real-world studies, ocrelizumab continues to represent a generally well-tolerated, high-efficacy DMT for relapsing forms of MS and a valuable pharmacotherapy for delaying disease progression in patients with PPMS. Of particular pertinence during a pandemic, ocrelizumab offers patients with MS the convenience of short ( $\approx 2$-hour), half-yearly infusions.

Data Selection Ocrelizumab: 716 records identified

Duplicates removed

214

Excluded during initial screening (e.g. press releases; news reports; not relevant drug/indication; preclinical study; reviews; case reports; not randomized trial)

Excluded during writing (e.g. reviews; duplicate data; small patient number; nonrandomized/phase I/II trials)

Cited efficacy/tolerability articles

Search Strategy: EMBASE, MEDLINE and PubMed from 2018 to present. Previous Adis Drug Evaluation published in 2018 was hand-searched for relevant data. Clinical trial registries/ databases and websites were also searched for relevant data. Key words were ocrelizumab, Ocrevus, primary progressive multiple sclerosis, relapsing multiple sclerosis. Records were limited to those in English language. Searches last updated 13 Jan 2021. 
Supplementary Information The online version contains supplementary material available at https://doi.org/10.1007/s40265-022-01672-9.

Acknowledgements During the peer review process, the manufacturer of ocrelizumab was also offered an opportunity to review this article. Changes resulting from comments received were made on the basis of scientific and editorial merit.

\section{Declarations}

Funding The preparation of this review was not supported by any external funding.

Authorship and Conflict of interest Yvette Lamb is a salaried employee of Adis International Ltd/Springer Nature, and declares no relevant conflicts of interest. All authors contributed to the review and are responsible for the article content.

Ethics approval, Consent to participate, Consent to publish, Availability of data and material, Code availability Not applicable.

\section{References}

1. Dobson R, Giovannoni G. Multiple sclerosis - a review. Eur J Neurol. 2019;26(1):27-40.

2. Klineova S, Lublin FD. Clinical course of multiple sclerosis. Cold Spring Harb Perspect Med. 2018;8:a028928.

3. Montalban X, Gold R, Thompson AJ, et al. ECTRIMS/EAN guideline on the pharmacological treatment of people with multiple sclerosis. Mult Scler J. 2018;24(2):96-120.

4. Hauser SL, Cree BAC. Treatment of multiple sclerosis: a review. Am J Med. 2020;133:1380-90.

5. Sabatino JJ Jr, Zamvil SS, Hauser SL. B-cell therapies in multiple sclerosis. Cold Spring Harb Perspect Med. 2019;9(2):01.

6. Milo R. Therapies for multiple sclerosis targeting B cells. Croat Med J. 2019;60(2):87-98.

7. Genentech. Ocrevus (ocrelizumab): US highlights of prescribing information. 2020. https://www.gene.com/. Accessed 13 Jan 2021.

8. Genentech. Ocrevus (ocrelizumab): EU summary of product characteristics. 2021. https://www.ema.europa.eu/. Accessed 13 Jan 2021.

9. Syed YY. Ocrelizumab: a review in multiple sclerosis. CNS Drugs. 2018;32(9):883-90.

10. Hauser SL, Bar-Or A, Comi G, et al. Ocrelizumab versus interferon beta-1a in relapsing multiple sclerosis. N Eng J Med. 2017;376(3):221-34.

11. Laplaud D, Lebrun-Frenay C, Vukusic S, et al. Assessing efficacy and safety of ocrelizumab in active relapsing multiple sclerosis in a pragmatic setting: PRO-MSACTIVE phase IV study interim analysis [ePresentation no. A-21-00271]. In: 7th Congress of the European Academy of Neurology (EAN). 2021.

12. Vollmer T, Freedman MS, Killestein J, et al. Recently diagnosed early-stage RRMS: NEDA, ARR, disability progression, serum neurofilament and safety: 1-year interim data from the ocrelizumab phase IIIb ENSEMBLE study [abstract no. 2261]. Neurology. 2021;96 (15 Suppl).

13. Smoot K, Gervasi-Follmar T, Chen C, et al. Evaluating the efficacy and safety of transitioning patients from natalizumab to ocrelizumab (OCTAVE). Mult Scler J. 2020;26(3 Suppl):280.

14. Weinstock-Guttman B, Bermel R, Cutter G, et al. Ocrelizumab treatment for relapsing-remitting multiple sclerosis after a suboptimal response to previous disease-modifying therapy: a nonrandomized controlled trial. Mult Scler J. 2021. https://doi. org/10.1177/13524585211035740.

15. Vermersch P, Oreja-Guevara C, Aksel S, et al. Efficacy and safety of ocrelizumab in patients with relapsing-remitting multiple sclerosis with suboptimal response to prior disease-modifying therapies: primary analysis from the phase $3 \mathrm{~b}$ CASTING single-arm, open-label trial. Eur J Neurol. 2021. https://doi.org/10.1111/ene. 15171.

16. Montalban X, Hauser SL, Kappos L, et al. Ocrelizumab versus placebo in primary progressive multiple sclerosis. N Engl J Med. 2017;376(3):209-20.

17. Turner B, Cree BAC, Kappos L, et al. Ocrelizumab efficacy in subgroups of patients with relapsing multiple sclerosis. J Neurol. 2019;266(5):1182-93.

18. Hauser SL, Kappos L, Arnold DL, et al. Five years of ocrelizumab in relapsing multiple sclerosis: OPERA studies open-label extension. Neurology. 2020;95(13):e1854-67.

19. Giovannoni G, Kappos L, De Seze J, et al. Long-term reduction of relapse rate and confirmed disability progression after 7.5 years of ocrelizumab treatment in patients with relapsing multiple sclerosis in the OPERA OLE [abstract no. P723]. Mult Scler J. 2021;27(2 Suppl):606-7.

20. Van Wijmeersch B, Comi G, Oreja-Guevara C, et al. Efficacy and safety of ocrelizumab in patients with RRMS with suboptimal response to prior disease-modifying therapies: 3-year data from CASTING and LIBERTO 1-year interim results [abstract no. P627]. Mult Scler J. 2021;27(2 Suppl):543-4.

21. Fox EJ, Markowitz C, Applebee A, et al. Ocrelizumab reduces progression of upper extremity impairment in patients with primary progressive multiple sclerosis: findings from the phase III randomized ORATORIO trial. Mult Scler. 2018;24(14):1862-70.

22. Wolinsky JS, Arnold DL, Brochet B, et al. Long-term follow-up from the ORATORIO trial of ocrelizumab for primary progressive multiple sclerosis: a post-hoc analysis from the ongoing openlabel extension of the randomised, placebo-controlled, phase 3 trial. Lancet Neurol. 2020;19(12):998-1009.

23. Butzkueven H, Spelman T, Horakova D, et al. Risk of requiring a wheelchair in primary progressive multiple sclerosis: data from the ORATORIO trial and the MSBase registry. Eur J Neurol. 2021. https://doi.org/10.1111/ene.14824.

24. Wolinksy JS, Vermersch P, Hartung H-P, et al. Sustained reduction in 48-week confirmed disability progression in patients with PPMS treated with ocrelizumab in the ORATORIO OLE: 8-year follow-up [abstract no. 158]. Mult Scler J. 2021;27:101-2.

25. Bhattacharyya S, Ali A, Bakshi R. Characterization of MRI activity following treatment with ocrelizumab for multiple sclerosis [abstract]. Mult Scler J. 2020;26(3 Suppl):382-3.

26. Braune S, Heer Y, Tozzi V, et al. Real-world experience with ocrelizumab in the German neurotransdata registry [abstract]. Mult Scler J. 2020;26(3 Suppl):69-70.

27. Buttmann M, Meuth S, Weber M, et al. Assessing the real-world effectiveness of ocrelizumab in patients with multiple sclerosisconfidence one-year interim analysis [abstract]. Mult Scler J. 2020;26(3 Suppl):517.

28. Butzkueven H, Spelman T, Ozakbas S, et al. Real-world experience with ocrelizumab in relapsing multiple sclerosis: insights from the MSOCR-R cohort, an MSBase registry sub-study [abstract no. 161]. Mult Scler J. 2021;27:104-6.

29. Engmann NJ, Sheinson D, Yang E, et al. Real-world outcomes with ocrelizumab compared with other disease-modifying therapies in US commercial claims databases [abstract no. 2911]. Neurology. 2020;94 (15 Suppl).

30. Pontieri L, Blinkenberg M, Bramow S, et al. Ocrelizumab treatment in multiple sclerosis: a Danish population-based cohort study. Eur J Neurol. 2021. https://doi.org/10.1111/ene.15142. 
31. Smoot K, Stuchiner T, Chen C, et al. Utilization, safety, and tolerability of ocrelizumab: year 3 data from the Providence ocrelizumab registry [abstract]. Mult Scler J. 2020;26(3 Suppl):319.

32. Vollmer B, Nair K, Sillau S, et al. Ocrelizumab real-world safety and effectiveness in the one year treatment of multiple sclerosis compared to other disease modifying therapies. Mult Scler J. 2019;25(Suppl 2):747-8.

33. Hartung HP, Berger T, Bermel RA, et al. Shorter infusion time of ocrelizumab: results from the randomized, double-blind ENSEMBLE PLUS substudy in patients with relapsing-remitting multiple sclerosis. Mult Scler Relat Disord. 2020;46:102492.

34. Vollmer TL, Cohen JA, Alvarez E, et al. Safety results of administering ocrelizumab per a shorter infusion protocol in patients with primary progressive and relapsing multiple sclerosis. Mult Scler Relat Disord. 2020;46:102454.

35. Buttmann M, Meuth $\mathrm{S}$, Weber $\mathrm{M}$, et al. Safety and tolerability in patients with multiple sclerosis receiving ocrelizumab in a realworld setting - confidence one-year interim analysis. Mult Scler J. 2020;26(3 Suppl):553-4.

36. Prockl V, Nickel FT, Utz KS, et al. Real world application of ocrelizumab in multiple sclerosis: single-center experience of 128 patients. J Neurol Sci. 2020;415:116973.

37. Hauser SL, Kappos L, Montalban X, et al. Safety of ocrelizumab in patients with relapsing and primary progressive multiple sclerosis. Neurology. 2021;97:e1546-59.

38. Mayer L, Kappos L, Racke MK, et al. Ocrelizumab infusion experience in patients with relapsing and primary progressive multiple sclerosis: results from the phase 3 randomized OPERA I, OPERA II, and ORATORIO studies. Mult Scler Relat Disord. 2019;30:236-43.

39. Bermel R, Waubant E, Pardo G, et al. Evaluation of shorter infusion times for ocrelizumab treatment in an extension substudy of the phase IIIb CHORDS trial. Mult Scler J. 2019;25(Suppl 2):779-80.

40. Hughes R, Whitley L, Fitovski K, et al. COVID-19 in ocrelizumab-treated people with multiple sclerosis. Mult Scler Relat Disord. 2021;49:102725.

41. Shin RK, Rammohan KW, Williams MJ. Expert perspectives on COVID-19 vaccination for people living with multiple sclerosis. Neurol Ther. 2021. https://doi.org/10.1007/s40120-021-00266-z.

42. Yamout BI, Zakaria M, Inshasi J, et al. MENACTRIMS practice guideline for COVID-19 vaccination in patients with multiple sclerosis. Mult Scler Relat Disord. 2021;56:103225.

43. Rae-Grant A, Day GS, Marrie RA, et al. Practice guideline recommendations summary: disease-modifying therapies for adults with multiple sclerosis. Neurology. 2018;90(17):777-88.

44. Giovannoni G, Airas L, Bove R, et al. Ocrelizumab treatment effect on upper limb function in PPMS patients with disability: subgroup results of the ORATORIO study to inform the ORATORIO-HAND study design [abstract no. P3.2-091]. Neurology. 2019;92 (15 Suppl).

45. Lucchetta RC, Tonin FS, Borba HHL, et al. Disease-modifying therapies for relapsing-remitting multiple sclerosis: a network meta-analysis. CNS Drugs. 2018;32(9):813-26.
46. McCool R, Wilson K, Arber M, et al. Systematic review and network meta-analysis comparing ocrelizumab with other treatments for relapsing multiple sclerosis. Mult Scler Relat Disord. 2019;29:55-61.

47. Samjoo IA, Worthington E, Drudge C, et al. Comparison of ofatumumab and other disease-modifying therapies for relapsing multiple sclerosis: a network meta-analysis. J Comp Eff Res. 2020;9(18):1255-74.

48. Siddiqui MK, Khurana IS, Budhia S, et al. Systematic literature review and network meta-analysis of cladribine tablets versus alternative disease-modifying treatments for relapsing-remitting multiple sclerosis. Curr Med Res Opin. 2018;34(8):1361-71.

49. National Institute for Health and Care Excellence. Ocrelizumab for treating relapsing-remitting multiple sclerosis. 2018. https:// www.nice.org.uk/. Accessed 13 Jan 2021.

50. Engmann NJ, Sheinson D, Bawa K, et al. Persistence and adherence to ocrelizumab compared with other disease-modifying therapies for multiple sclerosis in U.S. commercial claims data. J Manag Care Spec Pharm. 2021;27(5):639-49.

51. Rath L, Bui MV, Ellis J, et al. Fast and safe: optimising multiple sclerosis infusions during COVID-19 pandemic. Multiple Sclerosis and Related Disorders. 2021;47 (no pagination).

52. Zimmermann M, Brouwer E, Tice JA, et al. Disease-modifying therapies for relapsing-remitting and primary progressive multiple sclerosis: a cost-utility analysis. CNS Drugs. 2018;32(12):1145-57.

53. National Institute for Health and Care Excellence. Ocrelizumab for treating primary progressive multiple sclerosis. 2019. https:// www.nice.org.uk/. Accessed 13 Jan 2021.

54. Gibiansky E, Petry C, Mercier F, et al. Ocrelizumab in relapsing and primary progressive multiple sclerosis: Pharmacokinetic and pharmacodynamic analyses of OPERA I, OPERA II and ORATORIO. Br J Clin Pharmacol. 2020. https://doi.org/10.1111/bcp. 14658

55. Baker D, Pryce G, James LK, et al. The ocrelizumab phase II extension trial suggests the potential to improve the risk: benefit balance in multiple sclerosis. Mult Scler Relat Disord. 2020;44:102279.

56. Cross A, Bennett J, von Budingen HC, et al. Ocrelizumab treatment reduced levels of neurofilament light chain and numbers of $B$ cells in the cerebrospinal fluid of patients with relapsing multiple sclerosis in the OBOE study [abstract no. S56.008]. In: 71st Annual Meeting of the American Academy of Neurology. 2019.

57. Bar-Or A, Bennett J, Von Budingen H, et al. B cells, T cells and inflammatory CSF biomarkers in primary progressive MS and relapsing MS in the OBOE (ocrelizumab biomarker outcome evaluation) trial [abstract no. 1635]. Neurology. 2020;94 (15 Suppl).

58. MacMillan EL, Schubert JJ, Vavasour IM, et al. Magnetic resonance spectroscopy evidence for declining gliosis in MS patients treated with ocrelizumab versus interferon beta-1a. Mult Scler J Exp Transl Clin. 2019;5(4).

59. Bar-Or A, Calkwood JC, Chognot C, et al. Effect of ocrelizumab on vaccine responses in patients with multiple sclerosis: the VELOCE study. Neurology. 2020;95(14):e1999-2008. 